\title{
Measurement of the Multiplicity of Gluons Splitting to Bottom Quark Pairs in Hadronic $Z^{0}$ Decays
}

DELPHI Collaboration

\begin{abstract}
An inclusive measurement of the average multiplicity of $b \bar{b}$ pairs from gluons, $g_{b \bar{b}}$, in hadronic $Z^{0}$ events collected by the DELPHI experiment at LEP, is presented. A counting technique, based on jet $b$-tagging in 4 -jet events, has been used. Looking for secondary bottom production in events with production of any primary flavour, by requiring two $b$-tagged jets in well defined topological configurations, gave

$$
g_{b \bar{b}}=(0.21 \pm 0.11(\text { stat }) \pm 0.09(\text { syst })) \% .
$$

This result was checked with a different method designed to select events with four $b$ quarks in the final state. Agreement within the errors was found.
\end{abstract}


P.Abreu $^{21}$, W.Adam ${ }^{49}$, T.Adye ${ }^{36}$, I.Ajinenko ${ }^{41}$, G.D.Alekseev ${ }^{16}$, R.Alemany ${ }^{48}$, P.P.Allport ${ }^{22}$, S.Almehed $^{24}$, U.Amaldi $^{9}$, S.Amato ${ }^{46}$, P.Andersson ${ }^{43}$, A.Andreazza ${ }^{9}$, P.Antilogus ${ }^{9}$, W-D.Apel ${ }^{17}$, B.Asman ${ }^{43}$, J-E.Augustin ${ }^{25}$, A.Augustinus $^{30}$, P.Baillon ${ }^{9}$, P.Bambade ${ }^{19}$, F.Barao $^{21}$, M.Barbi ${ }^{46}$, G.Barbiellini ${ }^{45}$, D.Y.Bardin ${ }^{16}$, G.Barker ${ }^{9}$,

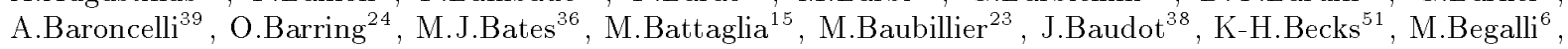
P.Beilliere $^{8}$, Yu.Belokopytov ${ }^{9,52}$, K.Belous ${ }^{41}$, A.C.Benvenuti ${ }^{5}$, M.Berggren ${ }^{46}$, D.Bertini ${ }^{25}$, D.Bertrand ${ }^{2}$, M.Besancon ${ }^{38}$, F.Bianchi ${ }^{44}$, M.Bigi ${ }^{44}$, M.S.Bilenky ${ }^{16}$, P.Billoir ${ }^{23}$, M-A.Bizouard ${ }^{19}$, D.Bloch ${ }^{10}$, M.Blume $^{51}$, M.Bonesini $^{27}$, W.Bonivento ${ }^{27}$, P.S.L.Booth ${ }^{22}$, A.W.Borgland ${ }^{4}$, G.Borisov ${ }^{38,41}$, C.Bosio ${ }^{39}$, O.Botner ${ }^{47}$, E.Boudinov $^{30}$, B.Bouquet ${ }^{19}$, C.Bourdarios ${ }^{19}$, T.J.V.Bowcock ${ }^{22}$, M.Bozzo $^{13}$, P.Branchini ${ }^{39}$, K.D.Brand ${ }^{35}$, T.Brenke $^{51}$, R.A.Brenner ${ }^{47}$, C.Bricman ${ }^{2}$, R.C.A.Brown ${ }^{9}$, P.Bruckman ${ }^{18}$, J-M.Brunet ${ }^{8}$, L.Bugge ${ }^{32}$, T.Buran ${ }^{32}$, T.Burgsmueller ${ }^{51}$, P.Buschmann ${ }^{51}$, S.Cabrera ${ }^{48}$, M.Caccia ${ }^{27}$, M.Calvi ${ }^{27}$, A.J.Camacho Rozas ${ }^{40}$, T.Camporesi ${ }^{9}$, V.Canale ${ }^{37}$, M.Canepa ${ }^{13}$, F.Cao ${ }^{2}$, F.Carena ${ }^{9}$, L.Carroll ${ }^{22}$, C.Caso ${ }^{13}$, M.V.Castillo Gimenez ${ }^{48}$, A.Cattai ${ }^{9}$, F.R.Cavallo ${ }^{5}$, V.Chabaud ${ }^{9}$, Ph.Charpentier ${ }^{9}$, L.Chaussard ${ }^{25}$, P.Chechia ${ }^{35}$, G.A.Chelkov ${ }^{16}$, M.Chen ${ }^{2}$, R.Chierici $^{44}$, P.Chliapnikov ${ }^{41}$, P.Chochula ${ }^{7}$, V.Chorowicz ${ }^{25}$, V.Cindro ${ }^{42}$, P.Collins ${ }^{9}$, R.Contri ${ }^{13}$, E.Cortina ${ }^{48}$, G.Cosme $^{19}$, F.Cossutti ${ }^{45}$, J-H.Cowell ${ }^{22}$, H.B.Crawley ${ }^{1}$, D.Crennell ${ }^{36}$, G.Crosetti ${ }^{13}$, J.Cuevas Maestro ${ }^{33}$, S.Czellar ${ }^{15}$, J.Dahm ${ }^{51}$, B.Dalmagne ${ }^{19}$, M.Dam ${ }^{28}$, G.Damgaard ${ }^{28}$, P.D.Dauncey ${ }^{36}$, M.Davenport ${ }^{9}$, W.Da Silva ${ }^{23}$, A.Deghorain $^{2}$, G.Della Ricca ${ }^{45}$, P.Delpierre ${ }^{26}$, N.Demaria ${ }^{34}$, A.De Angelis ${ }^{9}$, W.De Boer ${ }^{17}$, S.De Brabandere ${ }^{2}$, C.De Clercq ${ }^{2}$, C.De La Vaissiere ${ }^{23}$, B.De Lotto ${ }^{45}$, A.De Min ${ }^{35}$, L.De Paula ${ }^{46}$, H.Dijkstra ${ }^{9}$, L.Di Ciaccio ${ }^{37}$, A.Di Diodato $^{37}$, A.Djannati ${ }^{8}$, J.Dolbeau ${ }^{8}$, K.Doroba $^{50}$, M.Dracos $^{10}$, J.Drees ${ }^{51}$, K.-A.Drees ${ }^{51}$, M.Dris ${ }^{31}$, J-D.Durand $^{25,9}$, D.Edsall ${ }^{1}$, R.Ehret ${ }^{17}$, G.Eigen ${ }^{4}$, T.Ekelof ${ }^{47}$, G.Ekspong ${ }^{43}$, M.Elsing ${ }^{9}$, J-P.Engel ${ }^{10}$, B.Erzen ${ }^{42}$, M.Espirito Santo ${ }^{21}$, E.Falk ${ }^{24}$, G.Fanourakis ${ }^{11}$, D.Fassouliotis ${ }^{45}$, M.Feindt ${ }^{9}$, P.Ferrari ${ }^{27}$, A.Ferrer ${ }^{48}$, S.Fichet $^{23}$, T.A.Filippas ${ }^{31}$, A.Firestone ${ }^{1}$, P.-A.Fischer ${ }^{10}$, H.Foeth ${ }^{9}$, E.Fokitis ${ }^{31}$, F.Fontanelli ${ }^{13}$, F.Formenti ${ }^{9}$, B.Franek ${ }^{36}$, A.G.Frodesen $^{4}$, R.Fruhwirth ${ }^{49}$, F.Fulda-Quenzer ${ }^{19}$, J.Fuster ${ }^{48}$, A.Galloni ${ }^{22}$, D.Gamba ${ }^{44}$, M.Gandelman ${ }^{46}$, C.Garcia $^{48}$, J.Garcia ${ }^{40}$, C.Gaspar ${ }^{9}$, U.Gasparini ${ }^{35}$, Ph.Gavillet ${ }^{9}$, E.N.Gazis ${ }^{31}$, D.Gele ${ }^{10}$, J-P.Gerber $^{10}$, L.Gerdyukov $^{41}$, R.Gokieli ${ }^{50}$, B.Golob ${ }^{42}$, P.Goncalves ${ }^{21}$, G.Gopal ${ }^{36}$, L.Gorn ${ }^{1}$, M.Gorski ${ }^{50}$, Yu.Gouz ${ }^{44,52}$, V.Gracco ${ }^{13}$, E.Graziani ${ }^{39}$, C.Green ${ }^{22}$, A.Grefrath ${ }^{51}$, P.Gris ${ }^{38}$, G.Grosdidier ${ }^{19}$, K.Grzelak ${ }^{50}$, S.Gumenyuk ${ }^{41}$, M.Gunther ${ }^{47}$, J.Guy ${ }^{36}$, F.Hahn ${ }^{9}$, S.Hahn ${ }^{51}$, Z.Hajduk ${ }^{18}$, A.Hallgren ${ }^{47}$, K.Hamacher ${ }^{51}$, F.J.Harris ${ }^{34}$, V.Hedberg ${ }^{24}$, R.Henriques ${ }^{21}$, J.J.Hernandez ${ }^{48}$, P.Herquet ${ }^{2}$, H.Herr ${ }^{9}$, T.L.Hessing ${ }^{34}$, J.-M.Heuser ${ }^{51}$, E.Higon ${ }^{48}$, S-O.Holmgren ${ }^{43}$, P.J.Holt ${ }^{34}$, D.Holthuizen ${ }^{30}$, S.Hoorelbeke ${ }^{2}$, M.Houlden ${ }^{22}$, J.Hrubec ${ }^{49}$, K.Huet ${ }^{2}$, K.Hultqvist ${ }^{43}$, J.N.Jackson ${ }^{22}$, R.Jacobsson ${ }^{43}$, P.Jalocha ${ }^{9}$, R.Janik ${ }^{7}$, Ch.Jarlskog ${ }^{24}$, G.Jarlskog ${ }^{24}$, P.Jarry ${ }^{38}$, B.Jean-Marie ${ }^{19}$, E.K.Johansson ${ }^{43}$, L.Jonsson ${ }^{24}$, P.Jonsson ${ }^{24}$, C.Joram ${ }^{9}$, P.Juillot ${ }^{10}$, M.Kaiser ${ }^{17}$, F.Kapusta ${ }^{23}$, K.Karafasoulis ${ }^{11}$, M.Karlsson $^{43}$, S.Katsanevas ${ }^{25}$, E.C.Katsoufis ${ }^{31}$, R.Keranen ${ }^{4}$, Yu.Khokhlov ${ }^{41}$, B.A.Khomenko ${ }^{16}$, N.N.Khovanski ${ }^{16}$, B.King ${ }^{22}$, N.J.Kjaer ${ }^{30}$, O.Klapp ${ }^{51}$, H.Klein ${ }^{9}$, P.Kluit ${ }^{30}$, D.Knoblauch ${ }^{17}$, P.Kokkinias ${ }^{11}$, A.Konopliannikov ${ }^{41}$, M.Koratzinos ${ }^{9}$, K.Korcyl ${ }^{18}$, V.Kostioukhine ${ }^{41}$, C.Kourkoumelis ${ }^{3}$, O.Kouznetsov ${ }^{13,16}{ }^{\text {, }}$ M.Krammer $^{49}$, C.Kreuter ${ }^{9}$, I.Kronkvist ${ }^{24}$, J.Krstic ${ }^{11}, \quad$ Z.Krumstein $^{16}$, W.Krupinski ${ }^{18}, \quad$ P.Kubinec $^{7}$, W.Kucewicz ${ }^{18}$, K.Kurvinen ${ }^{15}$, C.Lacasta $^{9}$, I.Laktineh ${ }^{25}$, J.W.Lamsa ${ }^{1}$, L.Lanceri ${ }^{45}$, D.W.Lane ${ }^{1}$, P.Langefeld ${ }^{51}$, J-P.Laugier $^{38}$, R.Lauhakangas ${ }^{15}$, G.Leder ${ }^{49}$, F.Ledroit ${ }^{14}$, V.Lefebure ${ }^{2}$, C.K.Legan ${ }^{1}$, A.Leisos ${ }^{11}$, R.Leitner ${ }^{29}$, J.Lemonne $^{2}$, G.Lenzen ${ }^{51}$, V.Lepeltier ${ }^{19}$, T.Lesiak ${ }^{18}$, J.Libby ${ }^{34}$, D.Liko ${ }^{9}$, A.Lipniacka ${ }^{43}$, I.Lippi $^{35}$, B.Loerstad $^{24}$, J.G.Loken $^{34}$, J.M.Lopez ${ }^{40}$, D.Loukas ${ }^{11}$, P.Lutz ${ }^{38}$, L.Lyons ${ }^{34}$, J.MacNaughton ${ }^{49}$, G.Maehlum ${ }^{17}$, J.R.Mahon ${ }^{6}$, A.Maio $^{21}$, T.G.M.Malmgren ${ }^{43}$, V.Malychev ${ }^{16}$, F.Mandl ${ }^{49}$, J.Marco ${ }^{40}$, R.Marco ${ }^{40}$, B.Marechal ${ }^{46}$, M.Margoni $^{35}$, J-C.Marin $^{9}$, C.Mariotti ${ }^{9}$, A.Markou ${ }^{11}$, C.Martinez-Rivero ${ }^{33}$, F.Martinez-Vidal ${ }^{48}$, S.Marti i Garcia ${ }^{22}$, F.Matorras $^{40}$, C.Matteuzzi ${ }^{27}$, G.Matthiae ${ }^{37}$, M.Mazzucato ${ }^{35}$, M.Mc Cubbin ${ }^{22}$, R.Mc Kay ${ }^{1}$, R.Mc Nulty ${ }^{9}$, J.Medbo $^{47}$, C.Meroni ${ }^{27}$, S.Meyer ${ }^{17}$, W.T.Meyer ${ }^{1}$, M.Michelotto ${ }^{35}$, E.Migliore ${ }^{44}$, L.Mirabito ${ }^{25}$, W.A.Mitaroff ${ }^{49}$, U.Mjoernmark $^{24}$, T.Moa ${ }^{43}$, R.Moeller ${ }^{28}$, K.Moenig ${ }^{9}$, M.R.Monge ${ }^{13}$, P.Morettini ${ }^{13}$, H.Mueller ${ }^{17}$, K.Muenich $^{51}$, M.Mulders $^{30}$, L.M.Mundim ${ }^{6}$, W.J.Murray ${ }^{36}$, B.Muryn ${ }^{14,18}$, G.Myatt ${ }^{34}$, T.Myklebust ${ }^{32}$, F.Naraghi ${ }^{14}$, F.L.Navarria ${ }^{5}$, S.Navas ${ }^{48}$, K.Nawrocki ${ }^{50}$, P.Negri ${ }^{27}$, S.Nemecek ${ }^{12}$, W.Neumann ${ }^{51}$, N.Neumeister ${ }^{49}$, R.Nicolaidou $^{3}$, B.S.Nielsen ${ }^{28}$, M.Nieuwenhuizen $^{30}$, V.Nikolaenko ${ }^{10}$, M.Nikolenko $^{10,16}$, P.Niss $^{43}$, A.Nomerotski $^{35}$, A.Normand ${ }^{34}$, M.Novak ${ }^{12}$, W.Oberschulte-Beckmann ${ }^{17}$, V.Obraztsov ${ }^{41}$, A.G.Olshevski ${ }^{16}$, R.Orava ${ }^{15}$, G.Orazi $^{10}$, K.Osterberg ${ }^{15}$, A.Ouraou ${ }^{38}$, P.Paganini ${ }^{19}$, M.Paganoni ${ }^{9,27}$, R.Pain ${ }^{23}$, H.Palka ${ }^{18}$, Th.D.Papadopoulou ${ }^{31}$, K.Papageorgiou ${ }^{11}$, L.Pape ${ }^{9}$, C.Parkes ${ }^{34}$, F.Parodi ${ }^{13}$, A.Passeri ${ }^{39}$, M.Pegoraro $^{35}$, L.Peralta $^{21}$, H.Pernegger ${ }^{49}$, M.Pernicka ${ }^{49}$, A.Perrotta ${ }^{5}$, C.Petridou ${ }^{45}$, A.Petrolini ${ }^{13}$, H.T.Phillips ${ }^{36}$, G.Piana $^{13}$, F.Pierre $^{38}$, M.Pimenta ${ }^{21}$, T.Podobnik ${ }^{42}$, O.Podobrin ${ }^{9}$, M.E.Pol ${ }^{6}$, G.Polok ${ }^{18}$, P.Poropat ${ }^{45}$, V.Pozdniakov $^{16}$, P.Privitera $^{37}$, N.Pukhaeva ${ }^{16}$, A.Pullia ${ }^{27}$, D.Radojicic ${ }^{34}$, S.Ragazzi ${ }^{27}$, H.Rahmani ${ }^{31}$, J.Rames ${ }^{12}$, P.N.Ratoff ${ }^{20}$, A.L.Read ${ }^{32}$, M.Reale ${ }^{51}$, P.Rebecchi ${ }^{9}$, N.G.Redaelli ${ }^{27}$, M.Regler ${ }^{49}$, D.Reid ${ }^{9}$, R.Reinhardt ${ }^{51}$, P.B.Renton ${ }^{34}$, L.K.Resvanis ${ }^{3}$, F.Richard ${ }^{19}$, J.Richardson ${ }^{22}$, J.Ridky ${ }^{12}$, G.Rinaudo ${ }^{44}$, O.Rohne ${ }^{32}$, A.Romero ${ }^{44}$, P.Ronchese ${ }^{35}$, L.Roos $^{23}$, E.I.Rosenberg ${ }^{1}$, P.Roudeau ${ }^{19}$, T.Rovelli ${ }^{5}$, V.Ruhlmann-Kleider ${ }^{38}$, A.Ruiz ${ }^{40}$, K.Rybicki ${ }^{18}$, H.Saarikko ${ }^{15}$, Y.Sacquin ${ }^{38}$, A.Sadovsky ${ }^{16}$, O.Sahr ${ }^{14}$, G.Sajot ${ }^{14}$, J.Salt ${ }^{48}$, M.Sannino ${ }^{13}$, H.Schneider $^{17}$, U.Schwickerath $^{17}$, M.A.E.Schyns ${ }^{51}$, G.Sciolla ${ }^{44}$, F.Scuri ${ }^{45}$, P.Seager ${ }^{20}$, Y.Sedykh ${ }^{16}$, A.M.Segar ${ }^{34}$, A.Seitz $^{17}$, R.Sekulin $^{36}$, L.Serbelloni ${ }^{37}$, R.C.Shellard ${ }^{6}$, P.Siegrist ${ }^{9,38}$, R.Silvestre ${ }^{38}$, F.Simonetto ${ }^{35}$, A.N.Sisakian $^{16}$, B.Sitar ${ }^{7}$, T.B.Skaali ${ }^{32}$, G.Smadja ${ }^{25}$, N.Smirnov ${ }^{41}$, O.Smirnova ${ }^{24}$, G.R.Smith ${ }^{36}$, A.Sokolov ${ }^{41}$, O.Solovianov ${ }^{41}$, R.Sosnowski ${ }^{50}$, D.Souza-Santos ${ }^{6}$, T.Spassov ${ }^{21}$, E.Spiriti ${ }^{39}$, P.Sponholz ${ }^{51}$, S.Squarcia ${ }^{13}$, D.Stampfer ${ }^{9}$, C.Stanescu $^{39}$, S.Stanic ${ }^{42}$, S.Stapnes ${ }^{32}$, I.Stavitski ${ }^{35}$, K.Stevenson ${ }^{34}$, A.Stocchi ${ }^{19}$, R.Strub ${ }^{10}$, B.Stugu ${ }^{4}$, M.Szczekowski ${ }^{50}$, M.Szeptycka ${ }^{50}$, T.Tabarelli ${ }^{27}$, J.P.Tavernet ${ }^{23}$, O.Tchikilev ${ }^{41}$, F.Tegenfeldt ${ }^{47}$, F.Terranova ${ }^{27}$ ', J.Thomas $^{34}$, A.Tilquin ${ }^{26}$, J.Timmermans ${ }^{30}$, L.G.Tkatchev ${ }^{16}$, T.Todorov $^{10}$, S.Todorova ${ }^{10}$, D.Z.Toet ${ }^{30}$, 


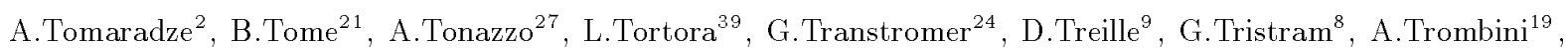
C.Troncon ${ }^{27}$, A.Tsirou ${ }^{9}$, M-L.Turluer ${ }^{38}$, I.A.Tyapkin ${ }^{16}$, M.Tyndel $^{36}$, S.Tzamarias ${ }^{11}$, B.Ueberschaer ${ }^{51}$, O.Ullaland ${ }^{9}$, V.Uvarov ${ }^{41}$, G.Valenti ${ }^{5}$, E.Vallazza ${ }^{45}$, C.Vander Velde ${ }^{2}$, G.W.Van Apeldoorn ${ }^{30}$, P.Van Dam ${ }^{30}$, W.K.Van Doninck ${ }^{2}$, J.Van Eldik ${ }^{30}$, A.Van Lysebetten ${ }^{2}$, N.Vassilopoulos ${ }^{34}$, G.Vegni ${ }^{27}$, L.Ventura ${ }^{35}$, W.Venus ${ }^{36}$, F.Verbeure $^{2}$, M.Verlato ${ }^{35}$, L.S.Vertogradov ${ }^{16}$, D.Vilanova ${ }^{38}$, P.Vincent ${ }^{25}$, L.Vitale ${ }^{45}$, A.S.Vodopyanov ${ }^{16}$, V.Vrba $^{12}$, H.Wahlen ${ }^{51}$, C.Walck ${ }^{43}$, C.Weiser ${ }^{17}$, A.M.Wetherell ${ }^{9}$, D.Wicke ${ }^{51}$, J.H.Wickens ${ }^{2}$, M.Wielers ${ }^{17}$, G.R.Wilkinson ${ }^{9}$, W.S.C.Williams ${ }^{34}$, M.Winter ${ }^{10}{ }$ M.Witek $^{18}$, T.Wlodek ${ }^{19}$, J.Yi ${ }^{1}$, K.Yip ${ }^{34}$, O.Yushchenko $^{41}{ }^{1}$ F.Zach $^{25}$, A.Zaitsev ${ }^{41}$, A.Zalewska ${ }^{9}, \quad$ P.Zalewski ${ }^{50}$, D.Zavrtanik ${ }^{42}$, E.Zevgolatakos ${ }^{11}, \quad$ N.I.Zimin ${ }^{16}$, G.C.Zucchelli ${ }^{43}$, G.Zumerle ${ }^{35}$

\footnotetext{
${ }^{1}$ Department of Physics and Astronomy, Towa State University, Ames IA 50011-3160, USA

${ }^{2}$ Physics Department, Univ. Instelling Antwerpen, Universiteitsplein 1, B-2610 Wilrijk, Belgium and IIHE, ULB-VUB, Pleinlaan 2, B-1050 Brussels, Belgium and Faculté des Sciences, Univ. de l'Etat Mons, Av. Maistriau 19, B-7000 Mons, Belgium

${ }^{3}$ Physics Laboratory, University of Athens, Solonos Str. 104, GR-10680 Athens, Greece ${ }^{4}$ Department of Physics, University of Bergen, Allégaten 55, N-5007 Bergen, Norway

${ }^{5}$ Dipartimento di Fisica, Università di Bologna and INFN, Via Irnerio 46, I-40126 Bologna, Italy ${ }^{6}$ Centro Brasileiro de Pesquisas Físicas, rua Xavier Sigaud 150, RJ-22290 Rio de Janeiro, Brazil and Depto. de Física, Pont. Univ. Católica, C.P. 38071 RJ-22453 Rio de Janeiro, Brazil and Inst. de Física, Univ. Estadual do Rio de Janeiro, rua São Francisco Xavier 524, Rio de Janeiro, Brazil ${ }^{7}$ Comenius University, Faculty of Mathematics and Physics, Mlynska Dolina, SK-84215 Bratislava, Slovakia

${ }^{8}$ Collège de France, Lab. de Physique Corpusculaire, IN2P3-CNRS, F-75231 Paris Cedex 05, France

${ }^{9}$ CERN, CH-1211 Geneva 23, Switzerland

${ }^{10}$ Centre de Recherche Nucléaire, IN2P3 - CNRS/ULP - BP20, F-67037 Strasbourg Cedex, France

${ }^{11}$ Institute of Nuclear Physics, N.C.S.R. Demokritos, P.O. Box 60228, GR-15310 Athens, Greece

${ }^{12}$ FZU, Inst. of Physics of the C.A.S. High Energy Physics Division, Na Slovance 2, 180 40, Praha 8, Czech Republic

${ }^{13}$ Dipartimento di Fisica, Università di Genova and INFN, Via Dodecaneso 33, I-16146 Genova, Italy

${ }^{14}$ Institut des Sciences Nucléaires, IN2P3-CNRS, Université de Grenoble 1, F-38026 Grenoble Cedex, France

${ }^{15}$ Helsinki Institute of Physics, HIP, P.O. Box 9, FIN-00014 Helsinki, Finland

${ }^{16}$ Joint Institute for Nuclear Research, Dubna, Head Post Office, P.O. Box 79, 101000 Moscow, Russian Federation

${ }^{17}$ Institut für Experimentelle Kernphysik, Universität Karlsruhe, Postfach 6980, D-76128 Karlsruhe, Germany

${ }^{18}$ Institute of Nuclear Physics and University of Mining and Metalurgy, Ul. Kawiory 26a, PL-30055 Krakow, Poland

${ }^{19}$ Université de Paris-Sud, Lab. de l'Accélérateur Linéaire, IN2P3-CNRS, Bât. 200, F-91405 Orsay Cedex, France

${ }^{20}$ School of Physics and Chemistry, University of Lancaster, Lancaster LA1 4YB, UK

${ }^{21}$ LIP, IST, FCUL - Av. Elias Garcia, 14-1 ${ }^{\circ}$, P-1000 Lisboa Codex, Portugal

${ }^{22}$ Department of Physics, University of Liverpool, P.O. Box 147, Liverpool L69 3BX, UK

${ }^{23}$ LPNHE, IN2P3-CNRS, Universités Paris VI et VII, Tour 33 (RdC), 4 place Jussieu, F-75252 Paris Cedex 05, France

${ }^{24}$ Department of Physics, University of Lund, Sölvegatan 14, S-22363 Lund, Sweden

${ }^{25}$ Université Claude Bernard de Lyon, IPNL, IN2P3-CNRS, F-69622 Villeurbanne Cedex, France

${ }^{26}$ Univ. d'Aix - Marseille II - CPP, IN2P3-CNRS, F-13288 Marseille Cedex 09, France

${ }^{27}$ Dipartimento di Fisica, Università di Milano and INFN, Via Celoria 16, I-20133 Milan, Italy

${ }^{28}$ Niels Bohr Institute, Blegdamsvej 17, DK-2100 Copenhagen 0, Denmark

${ }^{29} \mathrm{NC}$, Nuclear Centre of MFF, Charles University, Areal MFF, V Holesovickach 2, 180 00, Praha 8, Czech Republic

${ }^{30}$ NIKHEF, Postbus 41882, NL-1009 DB Amsterdam, The Netherlands

${ }^{31}$ National Technical University, Physics Department, Zografou Campus, GR-15773 Athens, Greece

${ }^{32}$ Physics Department, University of Oslo, Blindern, N-1000 Oslo 3, Norway

${ }^{33}$ Dpto. Fisica, Univ. Oviedo, Avda. Calvo Sotelo, S/N-33007 Oviedo, Spain, (CICYT-AEN96-1681)

${ }^{34}$ Department of Physics, University of Oxford, Keble Road, Oxford OX1 3RH, UK

${ }^{35}$ Dipartimento di Fisica, Università di Padova and INFN, Via Marzolo 8, I-35131 Padua, Italy

${ }^{36}$ Rutherford Appleton Laboratory, Chilton, Didcot OX11 OQX, UK

${ }^{37}$ Dipartimento di Fisica, Università di Roma II and INFN, Tor Vergata, I-00173 Rome, Italy

${ }^{38}$ CEA, DAPNIA/Service de Physique des Particules, CE-Saclay, F-91191 Gif-sur-Yvette Cedex, France

${ }^{39}$ Istituto Superiore di Sanità, Ist. Naz. di Fisica Nucl. (INFN), Viale Regina Elena 299, I-00161 Rome, Italy

${ }^{40}$ Instituto de Fisica de Cantabria (CSIC-UC), Avda. los Castros, S/N-39006 Santander, Spain, (CICYT-AEN96-1681)

${ }^{41}$ Inst. for High Energy Physics, Serpukov P.O. Box 35, Protvino, (Moscow Region), Russian Federation

${ }^{42}$ J. Stefan Institute, Jamova 39, SI-1000 Ljubljana, Slovenia and Department of Astroparticle Physics, School of Environmental Sciences, Kostanjeviska 16a, Nova Gorica, SI-5000 Slovenia, and Department of Physics, University of Ljubljana, SI-1000 Ljubljana, Slovenia

${ }^{43}$ Fysikum, Stockholm University, Box 6730, S-113 85 Stockholm, Sweden

${ }^{44}$ Dipartimento di Fisica Sperimentale, Università di Torino and INFN, Via P. Giuria 1, I-10125 Turin, Italy

${ }^{45}$ Dipartimento di Fisica, Università di Trieste and INFN, Via A. Valerio 2, I-34127 Trieste, Italy and Istituto di Fisica, Università di Udine, I-33100 Udine, Italy

${ }^{46}$ Univ. Federal do Rio de Janeiro, C.P. 68528 Cidade Univ., Tlha do Fundão BR-21945-970 Rio de Janeiro, Brazil

${ }^{47}$ Department of Radiation Sciences, University of Uppsala, P.O. Box 535, S-751 21 Uppsala, Sweden

${ }^{48}$ IFIC, Valencia-CSIC, and D.F.A.M.N., U. de Valencia, Avda. Dr. Moliner 50, E-46100 Burjassot (Valencia), Spain

${ }^{49}$ Institut für Hochenergiephysik, Österr. Akad. d. Wissensch., Nikolsdorfergasse 18, A-1050 Vienna, Austria

${ }^{50}$ Inst. Nuclear Studies and University of Warsaw, Ul. Hoza 69, PL-00681 Warsaw, Poland

${ }^{51}$ Fachbereich Physik, University of Wuppertal, Postfach 100 127, D-42097 Wuppertal, Germany

${ }^{52}$ On leave of absence from IHEP Serpukhov
} 


\section{Introduction}

The main contribution to the "gluon splitting" mechanism responsible for the secondary production of bottom quarks in $e^{+} e^{-}$annihilation, $g \rightarrow b \bar{b}$, is sketched in Fig. 1 . At lowest order, it consists of gluon radiation from a quark leg, followed by the gluon splitting into a $b \bar{b}$ pair. Interference with diagrams where a primary $b \bar{b}$ pair emits a gluon which splits into a $q \bar{q}$ quark pair is, to first order, non-zero only when $q \bar{q} \equiv b \bar{b}$ (four- $b$ events), and is small enough to be neglected [1].

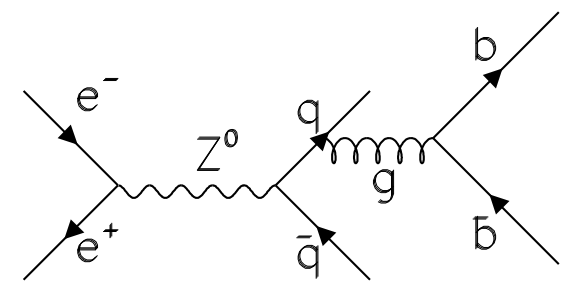

Figure 1: Lowest order contribution to the secondary production of bottom quark pairs (the symmetric graph with gluon radiation from the $\bar{q}$ line is implied).

The probability for secondary production of a bottom quark pair from a gluon per hadronic $Z^{0}$ decay,

$$
g_{b \bar{b}}=\frac{N\left(Z^{0} \rightarrow q \bar{q} g, g \rightarrow b \bar{b}\right)}{N\left(Z^{0} \rightarrow \text { hadrons }\right)}
$$

is expected to be very small, since the gluon must have sufficient energy to produce the bottom quark pair. The probability is an infrared finite quantity, because the quark mass provides a natural cutoff, so it can be safely computed in the framework of perturbative QCD [1-3]. However, large logarithmic terms, arising from the difference of the jet energy scale compared with the quark mass, can spoil the convergence of the perturbative expansion.

Knowledge of the probability of secondary production of $b \bar{b}$ pairs, $g_{b \bar{b}}$, is extremely important for the precision measurement of some electroweak quantities: for example, the uncertainty coming from $g_{b \bar{b}}$ is at present the biggest source of systematic error in the measurement of $R_{b}=\Gamma_{b \bar{b}} / \Gamma_{\text {had }}$ [4].

Recent theoretical calculations [1], performed at leading order in $\alpha_{S}$ with resummation of large leading and next-to-leading terms to all orders, predict the multiplicity of gluons splitting to $b \bar{b}$ pairs to be $g_{b \bar{b}}^{t h}=0.177 \%$, and that to $c \bar{c}$ pairs $g_{c \bar{c}}^{t h}=1.349 \%$. The JETSET Parton Shower (JETSET PS) Monte Carlo model [5], which provides a description of parton cascades accurate to leading logarithmic order and in agreement with the first order results for hard gluon production, predicts $g_{b \bar{b}}{ }^{P S}=0.16 \%$ and $g_{c \bar{c}} P S=1.7 \%$.

OPAL $[6,7]$ have recently measured $g_{c \bar{c}}$ to be $(2.27 \pm 0.28 \pm 0.41) \%[6]$ and observed the production of $\Upsilon$ mesons in $Z^{0}$ decay [8], which could also receive a contribution from the $g \rightarrow b \bar{b}$ mechanism.

In this analysis, about $1.4 \times 10^{6}$ hadronic $Z^{0}$ events collected by the DELPHI detector at LEP in 1994 have been used to measure the magnitude of the $g \rightarrow b \bar{b}$ effect. A $b$-tagging algorithm, based on lifetime information coming mainly from the vertex detector 
[9], was applied to jets in events showing a 4-jet topology. The presence of two identified $b$-jets in well defined topological configurations was required, in order to isolate $Z^{0} \rightarrow q \bar{q} g$ decays with subsequent $g \rightarrow b \bar{b}$ splitting. A second method was then developed, intended as a systematic check of the first one, in which the presence of at least $3 b$-tagged jets was required, thus looking for $g \rightarrow b \bar{b}$ events with primary $b \bar{b}$ flavour production and four $b$ quarks in the final state.

\section{The DELPHI detector and the hadronic event sample}

The DELPHI detector and its performance have been described in detail elsewhere $[10,11]$. Only the details most relevant to this analysis are mentioned here.

In the barrel region, the charged particle tracks are measured by a set of cylindrical tracking detectors whose axes are parallel to the $1.2 \mathrm{~T}$ solenoidal magnetic field and to the beam direction. The time projection chamber (TPC) is a cylinder with a length of $3 \mathrm{~m}$, an inner radius of $30 \mathrm{~cm}$ and an outer radius of $122 \mathrm{~cm}$. Between polar angles $\theta=39^{\circ}$ and $\theta=141^{\circ}$ with respect to the beam direction, tracks are reconstructed using up to 16 space points. Additional precise $R \Phi$ measurements, in the plane perpendicular to the magnetic field, are provided at larger and smaller radii by the Outer and Inner detectors respectively. The Outer Detector (OD) has five layers of drift cells at radii between 198 and $206 \mathrm{~cm}$ and covers polar angles from $42^{\circ}$ to $138^{\circ}$. The Inner Detector (ID) is a cylindrical drift chamber having an inner radius of $12 \mathrm{~cm}$ and outer radius of 28 $\mathrm{cm}$. When the data used in this analysis were taken, it covered polar angles between $29^{\circ}$ to $151^{\circ}$ and contained a jet chamber section providing $24 R \Phi$ coordinates surrounded by five layers of proportional chambers providing both $R \Phi$ and longitudinal $z$ coordinates.

The vertex detector (VD) is located between the LEP beam pipe and the ID. It consists of three concentric layers of silicon microstrip detectors placed at radii of $6.3,9$ and 11 $\mathrm{cm}$ from the interaction region. For all layers, the microstrip detectors provide hits in the $R \Phi$-plane with a measured resolution including alignment errors of about $8 \mu \mathrm{m}$. For the data taken in 1994, $z$ information was also available from the inner and the outer layer, due to an upgrade of the VD.

Only the data collected during 1994 were used for this analysis because the upgrade of the VD allowed the extension of the $b$-tagging algorithm to 3 dimensions, with a significant improvement in efficiency and purity with respect to the 2-dimensional case.

Hadronic decays of the $Z^{0}$ were selected by requiring the presence of 7 or more charged particle tracks satisfying the following requirements:

- measured momentum, $p$, greater than $0.2 \mathrm{GeV} / c$;

- polar angle in the range $20^{\circ}<\theta<160^{\circ}$;

- $\delta p / p<1$ where $\delta p$ is the error on $p$;

- track length greater than $30 \mathrm{~cm}$;

- impact parameter transverse to the beam $<4 \mathrm{~cm}$;

- impact parameter along the beam $<10 \mathrm{~cm}$.

Neutral particles, detected in the calorimeters surrounding the tracking chambers, were accepted if they had a reconstructed total energy greater than $0.5 \mathrm{GeV}$. These requirements selected $1.4 \times 10^{6}$ hadronic events.

The JETSET PS 7.4 Monte Carlo model [5] was used to generate simulated events. The Lund symmetric fragmentation function [5] was used to describe the hadronisation 
of the $u, d, s$ quarks, while the fragmentation of heavy quarks, $c$ and $b$, was parametrised by a Peterson function [12].

The response of the DELPHI detector to the generated events was simulated in full detail using the program DELSIM [11]. A sample of $4.5 \times 10^{6}$ simulated hadronic $Z^{0} \rightarrow q \bar{q}$ decays was used. An additional sample of $8 \times 10^{5} Z^{0} \rightarrow b \bar{b}$ events was used for the study of the background.

A further sample of $2.7 \times 10^{6} Z^{0} \rightarrow q \bar{q}$ events and two dedicated samples of $1.6 \times 10^{6}$ $Z^{0} \rightarrow b \bar{b}$ events and of $1.7 \times 10^{5} g \rightarrow b \bar{b}$ events were produced with the JETSET Matrix Element (ME) generator [5] and were used to check the model dependence of the result.

\section{Analysis method}

The major steps of the analysis were:

- the selection of 4-jet events from the previously mentioned hadronic sample;

- making a $b$-tagging requirement on two jets selected in a particular configuration, to select candidates originating from a $g \rightarrow b \bar{b}$ process;

- applying additional topological selections to improve the signal/background ratio;

- carrying out background subtraction and extracting the $g \rightarrow b \bar{b}$ signal.

Reconstructed charged and neutral particles were grouped into jets. Alternative jetfinding algorithms were investigated, such as LUCLUS [13] and JADE [14], but the DURHAM algorithm [15] was preferred as it gave the best agreement between data and simulation in the distribution of the number of jets. The value of the parameter $y_{c u t}$ was chosen by minimizing the final error (statistical plus systematic) on $g_{b \bar{b}}$. This involved a compromise between maximising the $g \rightarrow b \bar{b}$ purity of the 4-jet event sample, which increases with $y_{c u t}$, and maintaining the number of $g \rightarrow b \bar{b}$ 4-jet events selected, which falls with $y_{c u t}$ : the optimum was found to be $y_{\text {cut }}=0.017$.

Distributions of the number of jets in the event for data, for generic $q \bar{q}$ simulated events, and for simulated events containing $g \rightarrow b \bar{b}$ gluon splitting are shown in Fig. 2. The discrepancy between data and simulation in the fraction of 4-jet events amounts to

$$
\frac{f_{4-j e t}^{D A T A}}{f_{4-j e t}^{S I M}}=1.077 \pm 0.006
$$

According to the simulation, the efficiency of selecting 4-jet events with $g \rightarrow b \bar{b}$ is $(15.4 \pm 0.4) \%$, while the $g \rightarrow b \bar{b}$ content of the selected 4 -jet sample is $(1.17 \pm 0.03) \%$. The selected 4-jet data sample contained $3.2 \times 10^{4}$ events.

It was not possible to perform the $g_{b \bar{b}}$ measurement using the 3 -jet sample due to its very low $g \rightarrow b \bar{b}$ purity: with the value of $y_{\text {cut }}$ used, the $g \rightarrow b \bar{b}$ content in 3 -jet events was only $0.38 \%$.

Jets containing only one particle, or jets composed of neutral particles only, were rejected. Then the lifetime-signed impact parameters of charged particles with respect to the primary vertex in the event were used to tag $b$-jets, following a method originally developed by the ALEPH Collaboration [16] and adapted to the DELPHI data [9,11]. The impact parameters were considered in units of their errors, and a quantity $P_{j}$ was defined for each reconstructed jet in the event as the probability for the hypothesis that jet $j$ contained only charged tracks coming from the event primary vertex. After appropriate tuning of the errors [17], the distribution of the probability $P_{j}$ was essentially flat for light quark jets. Because of the significant lifetimes and decay multiplicities of charm and $b$ 
hadrons, it was then peaked at low values for charm quark jets, and strongly peaked at low values for $b$ quark jets, so $b$-tagging was implemented by selecting low values of $P_{j}$. Different selections on $P_{j}$ were used, depending on the analysis (see Sect. 4 and Sect. 5).

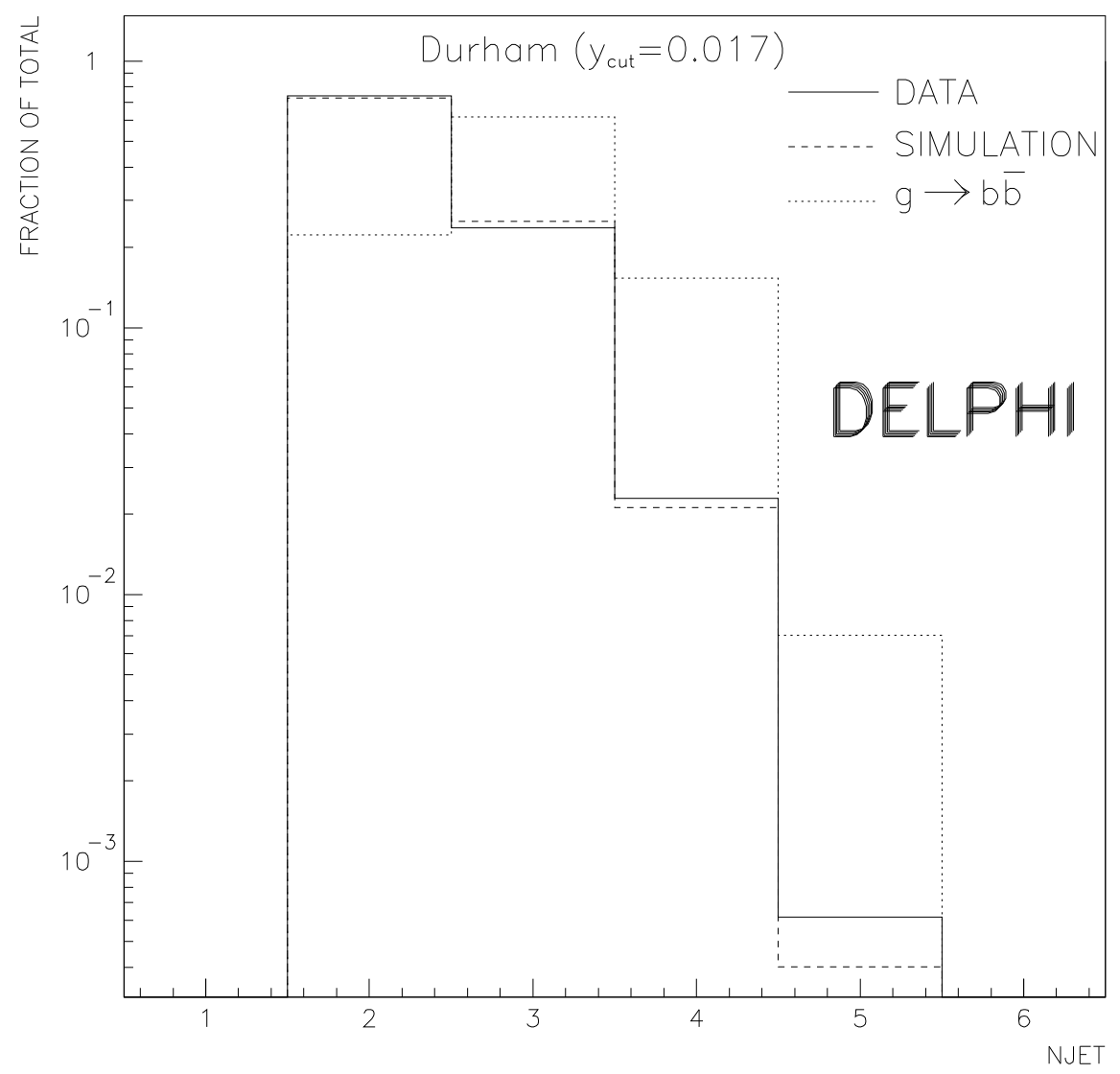

Figure 2: Comparison between data and simulation for the distribution of the number of jets in the event. The solid line represents the data, the dashed line represents the simulation, and the dotted line represents the shape of the distribution for $g \rightarrow b \bar{b}$ splitting events in the simulation, where $g \rightarrow b \bar{b}$ splitting events make up $0.16 \%$ of the total.

\section{Selection of $g \rightarrow b \bar{b}$ events and results}

The two jets forming the smallest angle in the event and satisfying the multiplicity requirements (see Sect. 3) in the 4-jet event sample were considered as candidates for originating from the gluon splitting process $g \rightarrow b \bar{b}$. The efficiency of this choice for selecting both of the two jets originating from a $g \rightarrow b \bar{b}$ process was estimated from the simulation to be $(54.4 \pm 1.3) \%$, while the corresponding probability of selecting only one of them was $(29.2 \pm 1.1) \%$. Other selections were studied, such as choosing the two least energetic jets, or the two jets forming the smallest invariant mass, but they resulted in lower efficiencies. 
The selected jets were labelled as jet 1 and jet 2, where jet 1 was more energetic than jet 2. The other two jets in the event were labelled as jets 3 and 4 , where jet 3 was more energetic than jet 4 .

The partons giving rise to jets 1 and 2 in simulated 4 -jet events which do not contain the process $g \rightarrow b \bar{b}$ (i.e. background events) are mainly gluons and light quarks: on average, $48 \%$ are gluons, $33 \%$ are light quarks, $9.5 \%$ are $b$-quarks, and $9.5 \%$ are charm quarks.

The effect of $b$-tagging on jets 1 and 2 was therefore studied. The ratio of the distributions of $P_{1}$ and $P_{2}$ probabilities between data and simulation in 4-jet events is shown in Fig. 3; good agreement can be observed. It was required that $P_{j}$ be less than 0.003 for both jets separately. This selection was chosen to minimise the final total error on $g_{b \bar{b}}$.

Jet tagging efficiencies and background evaluation were taken from simulation. They were checked by comparing the fraction of 4-jet events, in data and simulation, having at least one tagged jet or at least two tagged jets. The comparison of the single $b$-tagged jet fractions is shown in Table 1, which shows good agreement within statistical uncertainties.

Because of energy ordering, the single jet purities are different for jets 1 and 2: $\left.<\wp_{1}^{b}\right\rangle=(79.3 \pm 0.8) \%$ and $\left\langle\wp_{2}^{b}\right\rangle=(64.2 \pm 0.8) \%$. The efficiencies also differ. The mean jet $b$-tagging efficiencies and the mean jet $b$ purities averaged over jets 1 and 2 in 4-jet background events are $\left\langle\epsilon_{12}^{b}\right\rangle=(28.8 \pm 0.5) \%$ and $\left\langle\wp_{12}^{b}\right\rangle=(74.1 \pm 0.6) \%$, where $\left.<\wp_{12}^{b}\right\rangle$ is a weighted average using the single jet efficiencies as weights.

The $b$-tagging correlation between jet 1 and jet 2 was defined as $c_{12}^{b b}=\frac{\left\langle\epsilon_{12}^{b b}\right\rangle}{\left\langle\epsilon_{1}^{b}\right\rangle \times\left\langle\epsilon_{2}^{b}\right\rangle}-1$, where $\left\langle\epsilon_{1,2}^{b b}\right\rangle$ is the efficiency for simultaneous tagging of jet 1 and jet 2 , and $\left\langle\epsilon_{1}^{b}\right\rangle$ and $\left\langle\epsilon_{2}^{b}\right\rangle$ are the single jet $b$-tagging efficiencies. According to the simulation $c_{12}^{b b}=0.11 \pm 0.02$, while $c_{34}^{b b}=0.10 \pm 0.02$ (the errors are statistical).

\begin{tabular}{|c|c|}
\hline Jet & $f^{D A T A} / f^{S I M}$ \\
\hline \hline 1 & $1.04 \pm 0.03$ \\
2 & $1.02 \pm 0.04$ \\
3 & $1.00 \pm 0.03$ \\
4 & $1.02 \pm 0.04$ \\
\hline
\end{tabular}

Table 1: Ratio of fractions of events with at least one $b$-tagged jet in data and simulation 4-jet events.

The fractions of events with two $b$-tagged jets were then compared between data and simulation. In the case of jets 1 and 2 , i.e. the jets used in the $g_{b \bar{b}}$ measurement, this comparison gave

$$
\frac{f_{1,2}^{D A T A}}{f_{1,2}^{S I M}}=1.08 \pm 0.11
$$

According to the simulation, about $18 \%$ of the selected events contained the gluon splitting process $g \rightarrow b \bar{b}$.

The comparison of the fraction of events showing a $b$-tag on both jet 3 and jet 4 yielded

$$
\frac{f_{3,4}^{D A T A}}{f_{3,4}^{S I M}}=0.93 \pm 0.09
$$



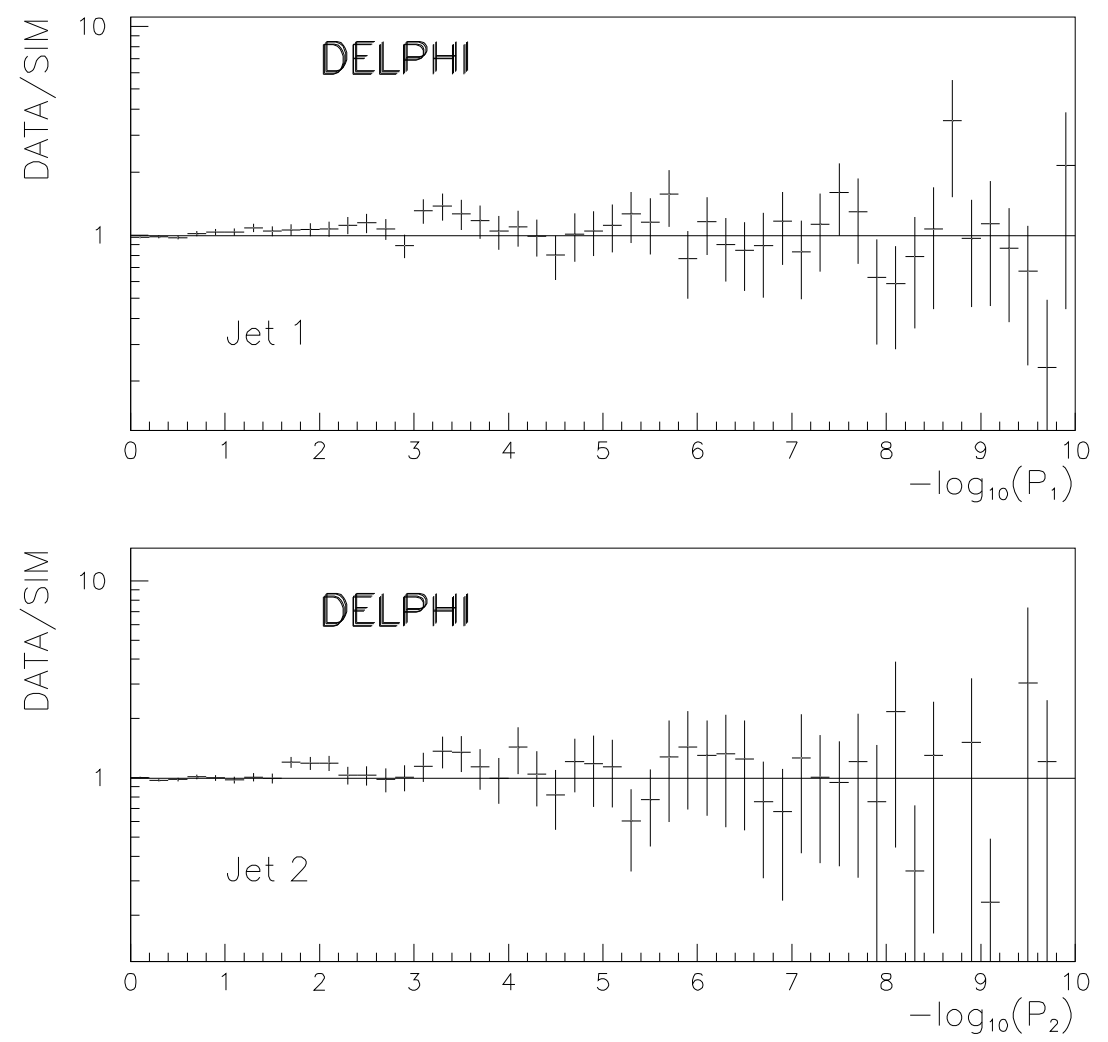

Figure 3: Ratio of the data and simulation $P_{1}$ and $P_{2}$ probability distributions for the two selected jets in 4 -jet events. The probabilities $P_{1}$ and $P_{2}$, used for $b$-tagging, are described in the text. 
with $3.5 \%$ of the selected simulated events containing the process $g \rightarrow b \bar{b}$. The mean jet $b$ purity averaged over jets 3 and 4 in the simulation is $(93 \pm 1) \%$. The result in Eq. (4) is thus essentially a data-simulation comparison of the product

$$
<\epsilon_{3}^{b}>\times<\epsilon_{4}^{b}>\times\left(1+c_{34}^{b b}\right)
$$

and was used to quote a systematic error from the $b$-tagging efficiency evaluation (see Sect. 5).

Applying the $b$-tag to jets 1 and 2 selected 90 events in the data, while the normalised number in the simulation was $16 \%$ lower (see Eq. (2) and Eq. (3)). Of the simulated sample, $(74.1 \pm 2.9) \%$ was background, $(18.3 \pm 2.5) \%$ contained $g \rightarrow b \bar{b}$ splitting, and $(7.6 \pm 1.7) \%$ contained $g \rightarrow c \bar{c}$ splitting.

Events with primary $b \bar{b}$ production constituted $(97.0 \pm 1.3) \%$ of the $(74.1 \pm 2.9) \%$ that were background, while $(2.4 \pm 1.2) \%$ were events with primary $c \bar{c}$ production and the remaining $(0.6 \pm 0.6) \%$ were light quark events. Most of the background came from $Z^{0} \rightarrow b \bar{b} g g$ events, in which at least one $b$ jet triggered the $b$-tag. Jets 1 and 2 , in such events, both arose from primary $b$ quarks in $50 \%$ of the cases, and from a primary $b$ quark and a gluon (wrongly tagged as a $b$ ) in $45 \%$ of the cases.

In the $(18.3 \pm 2.5) \%$ that were signal, $(42 \pm 8) \%$ were events with primary $b \bar{b}$ production, $(8 \pm 5) \%$ were with primary $c \bar{c}$ production, and $(50 \pm 8) \%$ were with primary light flavour production. The $Z^{0} \rightarrow b \bar{b}$ component is enhanced with respect to the other flavours since these events are four- $b$ events, so jets 1 and 2 are always $b$-jets, no matter how efficient the selection is.

Three further selections were then used to reduce the background.

Firstly, events in which jets 1 and 2 were the two most energetic ones were rejected. This requirement rejected $(3 \pm 2) \%$ of the signal and $(17 \pm 2) \%$ of the background, in the simulated tagged sample.

Secondly, the rapidity $\eta_{T 1}$ of jet 1 with respect to the thrust direction of the event was used to distinguish between primary and secondary $b$ production. Jet 1 , being the more energetic of the two selected and tagged jets and thus having probably lost less energy in radiative processes, has a higher probability of being close to the thrust direction if it is a primary $b$ jet from $Z^{0} \rightarrow b \bar{b}$ decay than if it is a secondary $b$ jet from $g \rightarrow b \bar{b}$. The rapidity $\eta_{T 1}$ is thus expected to be higher in $Z^{0} \rightarrow b \bar{b}$ background events than in $g \rightarrow b \bar{b}$ signal events. This effect is shown in Fig. 4a: the distributions were obtained for simulated untagged events in which jet 1 was known from the simulation to originate from a secondary $b$ quark $(g \rightarrow b \bar{b}$ process) or from a primary $b$ quark (background). Fig. $4 \mathrm{~b}$ represents the distributions of the same variable obtained after $b$-tagging of jets 1 and 2 for data, simulated background (mostly $Z^{0} \rightarrow b \bar{b}$ ), and simulated signal $(g \rightarrow b \bar{b})$. Events with $\eta_{T 1}<1.2$ were selected. This selection, applied to 4 -jet events satisfying the $b$-tag requirement on jets 1 and 2 , kept $(60 \pm 8) \%$ of the signal and $(32.7 \pm 2.7) \%$ of the background, according to the simulation. The presence of signal in the data distribution in Fig. 4b was then checked. A maximum likelihood fit of the shapes of the simulated signal and background components to the data distribution showed a signal of $(40 \pm 16) \%$ of the data sample. This fit result was not used in the $g_{b \bar{b}}$ measurement ${ }^{\dagger}$, but was considered as a consistency check.

Thirdly, the variable $\left|\cos \alpha_{1234}\right|$, where $\alpha_{1234}$ is the angle between the plane $\Pi_{12}$ formed by jets 1 and 2 and the plane $\Pi_{34}$ formed by jets 3 and 4 , i.e. $\alpha_{1234} \equiv \Pi_{12} \widehat{\Pi}_{34}$, was used to suppress the $b \bar{b} g g$ background. This variable is similar to the Bengtsson-Zerwas angle [18], but, in the original Bengtsson-Zerwas formulation, energy ordering of the four jets was

\footnotetext{
${ }^{\dagger}$ Since the 4-jet rates in data and simulation disagreed, the shape of the background in the simulation was considered insufficiently reliable to be used for this purpose.
} 
applied to separate primary from secondary jets (i.e. the variable was the angle between the plane spanned by the two most energetic jets in the event and the plane spanned by the two least energetic jets in the event). The Bengtsson-Zerwas angle is known to distinguish between $q \bar{q} q \bar{q}$ final states, as expected in signal $g \rightarrow b \bar{b}$ events $(q \bar{q} b \bar{b})$, and $q \bar{q} g g$ final states, especially in the cases in which the gluons come from a triple gluon vertex $g \rightarrow g g$ [18]. The radiated virtual gluon in the process $Z^{0} \rightarrow q \bar{q} g$ is polarized in the plane of the three-parton event, and this is reflected in its subsequent splitting, by strongly favouring $g \rightarrow q \bar{q}$ emission out of this plane compared to the $g \rightarrow g g$ process, which is favoured to happen in the event plane. Fig. 5a shows the distribution of $\left|\cos \alpha_{1234}\right|$ for simulated signal and $Z^{0} \rightarrow b \bar{b}$ background events for the untagged 4-jet sample, while Fig. 5b shows the same distributions after $b$-tagging of jets 1 and 2 for data, simulated background, and simulated signal $(g \rightarrow b \bar{b})$. Events with $\left|\cos \alpha_{1234}\right|>0.8$ were rejected. This selection, applied to 4-jet events satisfying the $b$-tag requirement on jets 1 and 2 , kept $(75 \pm 8) \%$ of the simulated signal and $(58.1 \pm 2.8) \%$ of the simulated background. The same fitting procedures as in the $\eta_{T 1}$ case were used in order to check the presence of signal in the data distribution, with compatible results.

The combined effect of these last three selections (referred to as "topological selections" in the following) was checked in data and simulation by comparing the fractions of events surviving the cuts in the untagged 4-jet sample (the tagged sample contained too high a fraction of signal events to provide a valid cross-check). A discrepancy

$$
\frac{f^{D A T A}}{f^{S I M}}=1.09 \pm 0.01
$$

was measured. This ratio was then used to correct the background evaluation (see below).

After all the selections, 22 events were left in data, while the normalised number in simulation was $21 \%$ lower. In the simulation, the background consisted solely of events with primary $b \bar{b}$ production. Thus it could be estimated more precisely using the additional sample of $8 \times 10^{5} Z^{0} \rightarrow b \bar{b}$ events. Including this sample, the simulated events were found to be distributed as follows: $(52.0 \pm 6.9) \%$ were background events, $(39.5 \pm 8.2) \%$ were signal events containing $g \rightarrow b \bar{b}$ gluon splitting, and $(8.5 \pm 3.8) \%$ were events containing $g \rightarrow c \bar{c}$ gluon splitting. The efficiency of the method in detecting simulated $g \rightarrow b \bar{b}$ events was computed to be

$$
\epsilon_{g \rightarrow b \bar{b}}=(0.31 \pm 0.06) \%
$$

where the error comes from the simulation statistics.

The simulated background events were scaled to the data taking into account a) the $7.7 \%$ data-simulation discrepancy in the 4-jet rate, Eq. (2), and b) the discrepancy found in Eq. (6). After this correction, the 22 data events were expected to contain $10.9 \pm 1.4$ background events, where the error is statistical. This scaling correction was not applied to events containing gluon splitting, assuming that JETSET PS was able to reproduce correctly the distribution of the number of jets and the topological distributions for this kind of event.

Using the OPAL $g_{c \bar{c}}$ measurement together with the probability of a $g \rightarrow c \bar{c}$ event being selected, taken from the simulation, $2.0 \pm 0.9 \mathrm{~g} \rightarrow c \bar{c}$ events were expected inside the data sample. The sample was thus estimated to contain $9.1 \pm 4.6 \pm 1.7 g \rightarrow b \bar{b}$ events, where the first error is the data statistical error and the second one comes from the simulation statistics and the $g \rightarrow c \bar{c}$ subtraction. Then, using Eq. (7) for the efficiency evaluation of the $g \rightarrow b \bar{b}$ signal, the result

$$
g_{b \bar{b}}=(0.21 \pm 0.11) \%
$$


was obtained, where the error comes from the data sample statistics.
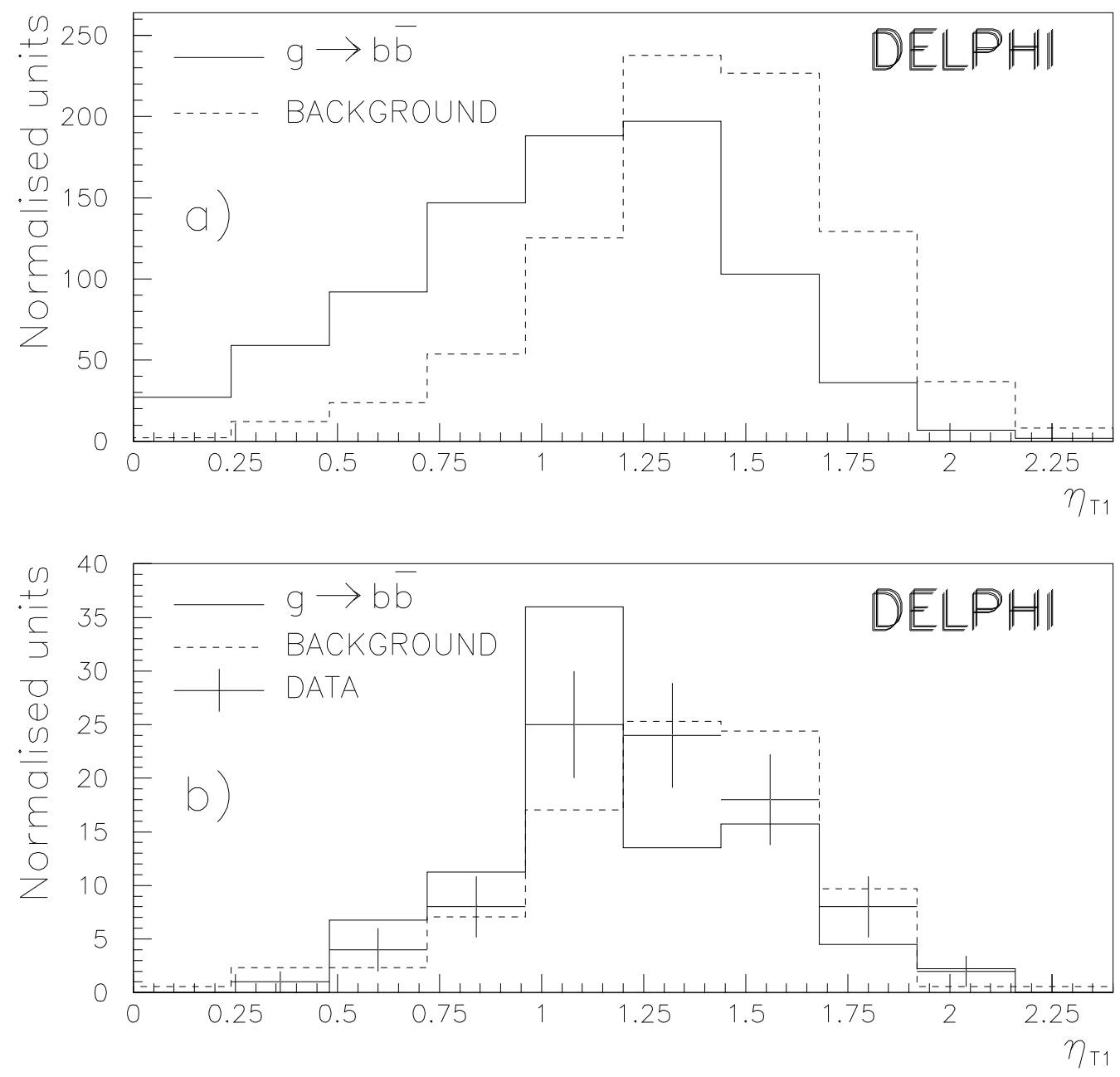

Figure 4: Distributions of the rapidity $\eta_{\mathrm{T} 1}$ with respect to the thrust direction of the more energetic of the two jets that formed the smallest angle in the event: a) simulated untagged sample, b) data and simulated tagged samples normalized to the data. Events with $\eta_{\mathrm{T} 1}$ above 1.2 were rejected.

\section{$5 \quad$ Estimation of systematic errors}

The first source of systematic uncertainty considered was the discrepancy in 4-jet rates between data and simulation, see Eq. (2). It was considered that the assumption that the 4-jet rate for events containing $g \rightarrow b \bar{b}$ splitting was well reproduced by JETSET PS could be wrong by the full amount of the discrepancy found in the case of the background, that is $7.7 \%$. This gave a systematic contribution of $\pm 0.02 \%$ to the $g_{b \bar{b}}$ measurement. 

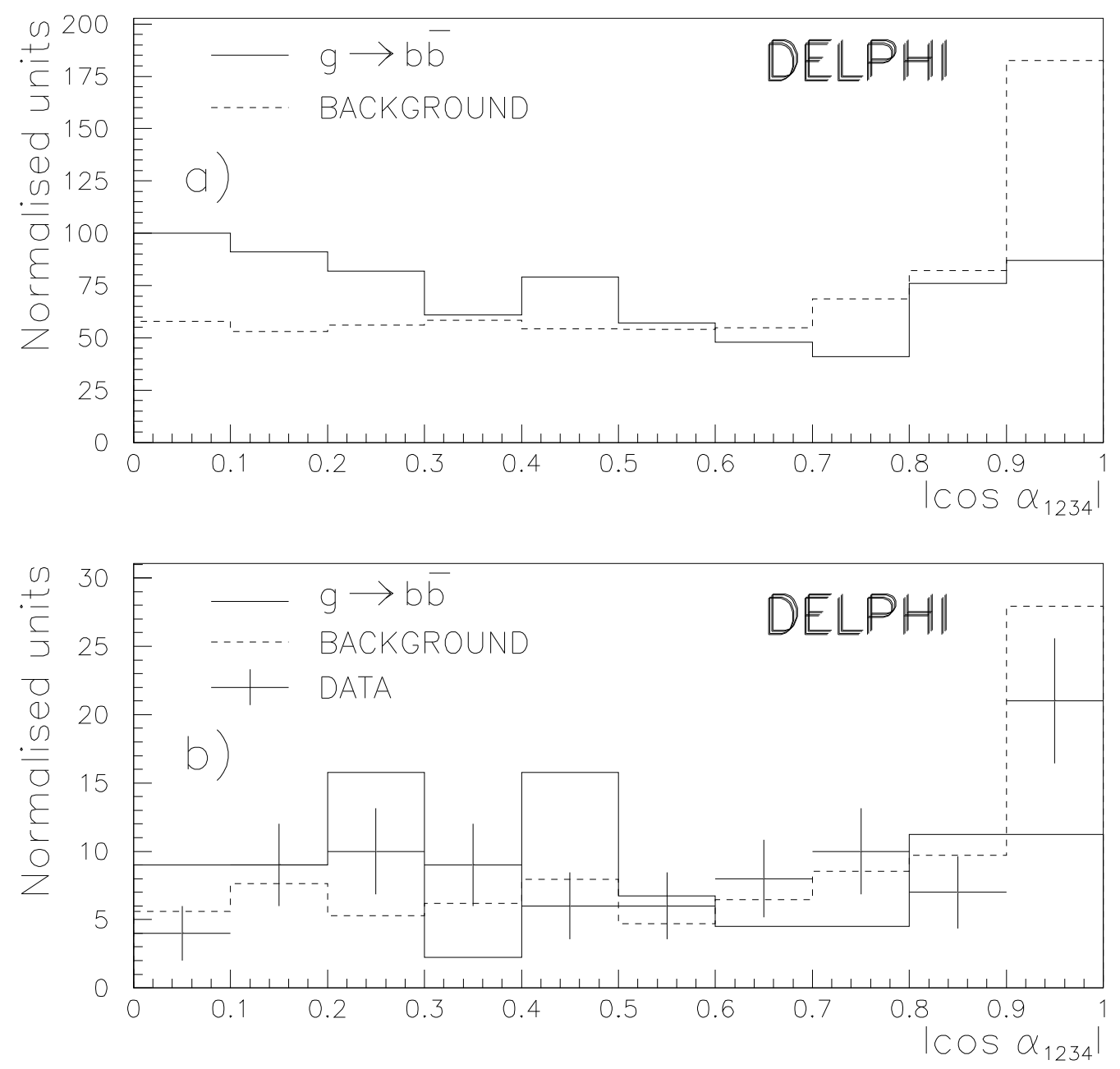

Figure 5: Distributions of $\left|\cos \alpha_{1234}\right|$ in 4-jet events, where $\alpha_{1234}$ is the angle formed by the two planes $\Pi_{12}$ and $\Pi_{34}$ spanned by jets 12 and 34: a) simulated untagged sample, b) data and simulated tagged samples normalized to the data. Events with $\left|\cos \alpha_{1234}\right|$ above 0.8 were rejected. 
The systematic uncertainty due to the particular choice of $y_{c u t}$ in the clusterization algorithm (see Sect. 3) was studied. The measurement was entirely repeated using a $y_{\text {cut }}$ value of 0.008 , which increased the statistical size of the selected sample, but decreased its $g \rightarrow b \bar{b}$ purity. Using the same selections as in the described measurement, the result

$$
g_{b \bar{b}}{ }^{0.008}=(0.20 \pm 0.08(\text { stat }) \pm 0.13(\text { syst })) \%
$$

was obtained. The statistical overlap with the $y_{\text {cut }}=0.017$ sample was about $50 \%$ of the latter. A contribution of $\pm 0.01 \%$ was thus assigned to $g_{b \bar{b}}$.

Another source of systematic uncertainty considered was the $b$-tagging efficiency. This affects both the signal extraction and the background estimate, the latter being totally composed of events with primary $b \bar{b}$ production. In order to evaluate the impact of this effect on the final measurement, Eq. (4), which compares $b$-tag performances on jets 3 and 4 (see Eq. (5)), was assumed to be also representative of the $b$-tag performances on jets 1 and 2. As the central value of the ratio in Eq. (4) is compatible with one, its error was used to evaluate the uncertainty with which the fraction of events having both jets 1 and 2 tagged as $b$ jets was known ${ }^{\ddagger}$. This procedure also takes into account the jet-jet $b$-tag efficiency correlations (see Eq. (5)) which, according to the simulation, are of the same order for jets 1 and 2 and for jets 3 and 4 ( $\operatorname{see} c_{12}^{b b}$ and $c_{34}^{b b}$ in Sect. 4). This gave a systematic contribution of $\pm 0.04 \%$ to the $g_{b \bar{b}}$ measurement.

Systematic uncertainties coming from the effect of cutting on distributions like the jet rapidity $\eta_{T 1}$ and the angle between jet planes $\alpha_{1234}$, and from the rejection of events in which jets 1 and 2 were the most energetic ones, were then taken into account. It was assumed that the uncertainty on the background evaluation coming from the discrepancy in Eq. (6) could be of the same order as the discrepancy itself. Furthermore, as the correction in Eq. (6) was not applied to the $g \rightarrow b \bar{b}$ signal, it was assumed that the signal evaluation could also be affected by the same uncertainty. This gave a systematic contribution of $\pm 0.04 \%$ to the $g_{b \bar{b}}$ measurement.

Uncertainties coming from the models used in the simulation were also estimated. The measurement assumes that the JETSET PS model correctly describes the features of the events containing $g \rightarrow b \bar{b}$ and the background events. This was checked using the sample of $2.7 \times 10^{6} Z^{0} \rightarrow q \bar{q}$ events and the special samples of $1.7 \times 10^{5} \mathrm{~g} \rightarrow b \bar{b}$ events and $1.6 \times 10^{6}$ $Z^{0} \rightarrow b \bar{b}$ events, generated with the JETSET Matrix Element [5] simulation program and analysed by the full DELPHI off-line reconstruction chain. The Matrix Element model is expected to give a quite different description of the $g \rightarrow b \bar{b}$ mechanism, as it implements the analytical 4-parton final state cross section calculation (second order QCD). Thus it is well suited to estimating a possible bias in the measurement. The measurement was therefore repeated using the Matrix Element sample (which reproduces the experimental 4-jet rate to better than $1 \%)$ as reference. The result was $g_{b \bar{b}}{ }^{M E}=(0.19 \pm 0.04) \%$, where the error comes from the ME simulation statistics. The larger of a) the difference between the measurements using the JETSET PS and JETSET ME models, and b) its statistical error, was assigned as the systematic uncertainty, i.e. $\pm 0.04 \%$.

In order to check the validity of this assumption in more detail, the uncertainty coming from the difference between the various simulation models in predicting the gluon energy spectrum was estimated separately (this contribution is included in the uncertainty estimated above from the $g_{b \bar{b}} M E$ result). Fig. 6a shows the energy distributions of the gluon before splitting to $b \bar{b}$ for the two models considered. The differential efficiency of the method as a function of the gluon energy in the JETSET ME simulation after all selections, computed using the large $g \rightarrow b \bar{b}$ dedicated sample, is shown in Fig. 6b. This

${ }^{\ddagger}$ Eq. (3) cannot be used for this purpose because that ratio contains the unknown fraction of gluon splitting events, whose measurement is the goal of the analysis. 
efficiency distribution was applied to the JETSET PS gluon spectrum and an integral efficiency was computed, which differed by $2.5 \%$ from the quantity in Eq. (7). Such a difference corresponds to a variation of less than $\pm 0.01 \%$ on $g_{b \bar{b}}$, which is compatible with the $\pm 0.04 \%$ uncertainty evaluated above.

The differential efficiency of the method is near zero in the first bin of the distribution shown in Fig. 6b, near the kinematical threshold for $g \rightarrow b \bar{b}$. A further systematic contribution was therefore computed as the difference, normalized to the area of the distributions, of the first bin populations of the JETSET PS and JETSET ME gluon energy spectra. This gave a contribution of $\pm 0.01 \%$ to $g_{b \bar{b}}$.

The effect of a $b$ quark mass different from the one used in the simulation was evaluated by making use, at the generator level, of the Accomando-Ballestrero-Maina WPHACT program [19], which is based on a matrix element calculation that includes quark masses in a general way. Events including the $g \rightarrow b \bar{b}$ process were generated for $b$ quark masses ranging from 4.7 to $5.3 \mathrm{GeV} / c^{2}$ and gluon spectra were obtained. The differential efficiency in Fig. 6 was then applied to these spectra, and the spread of the results was taken as a systematic uncertainty. This gave a contribution of $\pm 0.01 \%$ to $g_{b \bar{b}}$.

Another source of systematic error is related to the contribution of the $g \rightarrow c \bar{c}$ background. Varying the value of $g_{c \bar{c}}$ according to the error quoted by OPAL [6,7] gave a contribution of $\pm 0.01 \%$ to the $g_{b \bar{b}}$ measurement.

Finally, the errors arising from the uncertainties in the detection efficiencies and in the background subtractions due to the limited simulation statistics amounted to $\pm 0.06 \%$.

\begin{tabular}{|c|c|}
\hline Source & $\Delta g_{b \bar{b}}(\%)$ \\
\hline \hline 4-jet rate discrepancy & \pm 0.02 \\
$y_{\text {cut }}$ choice & \pm 0.01 \\
$b$-tag efficiency & \pm 0.04 \\
Topological cuts & \pm 0.04 \\
QCD Model (PS-ME) & \pm 0.04 \\
Threshold efficiency & \pm 0.01 \\
$b$ mass & \pm 0.01 \\
Value of $g \rightarrow c \bar{c}$ & \pm 0.01 \\
Sim. statistics & \pm 0.06 \\
\hline \hline Total & \pm 0.09 \\
\hline
\end{tabular}

Table 2: Summary of the systematic errors on the $g_{b \bar{b}}$ measurement.

Table 2 summarises the various contributions to the systematic error. The final estimated systematic error was thus $\pm 0.09 \%$, giving

$$
g_{b \bar{b}}=(0.21 \pm 0.11(\text { stat }) \pm 0.09(\text { syst })) \% \text {. }
$$

This result was checked by another method, designed to select a sample of candidate events with primary $b \bar{b}$ production radiating a hard gluon that split to $b \bar{b}$. In this method, events with four $b$ quarks in the final state were searched for. This was achieved by asking for 4 -jet events in which at least three of the jets were $b$-tagged. Looser $b$-tag selections were used, in order to counteract the statistical reduction of the sample: $P_{j}<0.005$ was required for each jet. Any jet showing a $b$-tag was allowed to participate in the counting 
procedure, but the same topological selections on the variables $\eta_{T 1}$ and $\alpha_{1234}$, as in Sect. 4, were applied, with the same jet labelling.

The probability of secondary bottom production in events with primary bottom flavour is expected to be the same as in primary light flavoured events, at least to the precision of the present measurement (see for example Table 1 in reference [1]).

After all the described selections, 13 events in data were left, with no statistical overlap with the 22 events sample of the previous method.

Using the JETSET PS model as reference yielded

$$
g_{b \bar{b}}=(0.29 \pm 0.16) \%
$$

where the error is statistical only. This value is compatible within the errors with the more precise result in Eq. (9).

\section{Conclusions}

An inclusive measurement of the average multiplicity of gluons splitting into $b \bar{b}$ pairs in hadronic $Z^{0}$ decays collected by the DELPHI experiment at LEP has been presented. The experimental result is:

$$
g_{b \bar{b}}=(0.21 \pm 0.11(\text { stat }) \pm 0.09(\text { syst })) \% .
$$

A check with a different method, looking for events with four $b$ quarks in the final state, gave a result compatible with this within its substantially larger errors.

This result is also compatible with theoretical expectations [1] and with the JETSET model prediction of $0.16 \%$.

\section{Acknowledgements}

We thank A. Ballestrero for providing us with the WPHACT program and a modified version of it dedicated to the study of the $g \rightarrow b \bar{b}$ process. We are greatly indebted to our technical collaborators and to the funding agencies for their support in building and operating the DELPHI detector, and to the members of the CERN-SL Division for the excellent performance of the LEP collider. 

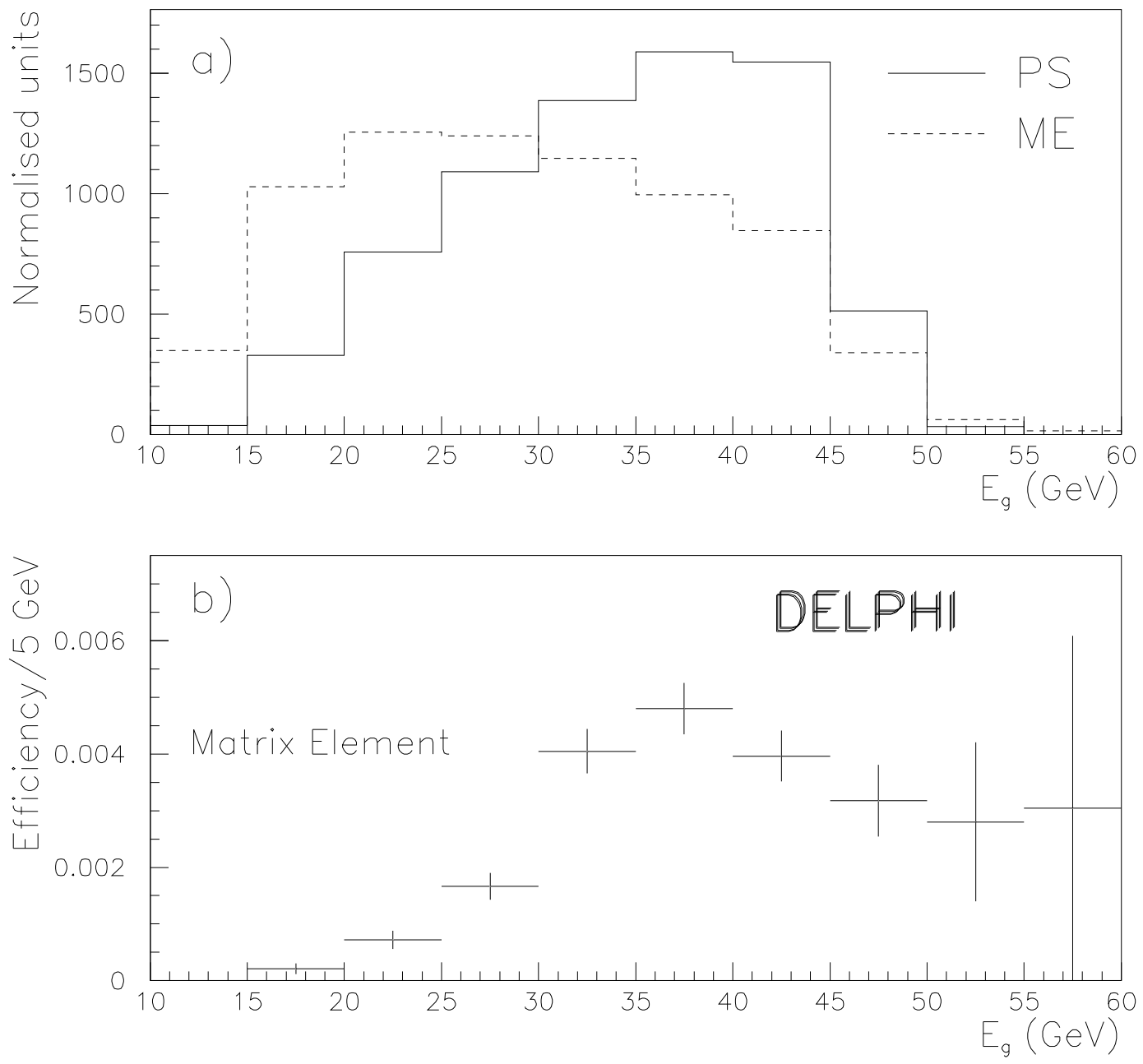

Figure 6: a) Energy distributions of gluons splitting to $b \bar{b}$ in the JETSET PS (solid line) and JETSET ME (dashed line) simulations. b) Differential efficiency of the method as a function of the energy of the splitting gluon (JETSET ME). 


\section{References}

[1] M.H. Seymour, Nucl. Phys. B436 (1995) 163.

[2] M.L. Mangano, P. Nason, Phys. Lett. B285 (1992) 160.

[3] M.H. Seymour, Z. Phys. C63 (1994) 99.

[4] The LEP Collaborations, "A Combination of Preliminary Electroweak Measurements and Constraints on the Standard Model", CERN-PPE/96-183, 1996.

[5] T. Sjöstrand, Comp. Phys. Comm. 39 (1986) 347;

T. Sjöstrand and M. Bengtsson, Comp. Phys. Comm. 43 (1987) 367;

T. Sjöstrand, JETSET 7.3 manual, preprint CERN-TH6488/92 (1992).

[6] OPAL Coll., R. Akers et al., Phys Lett. B353 (1995) 595.

[7] OPAL Coll., R. Akers et al., Z. Phys C67 (1995) 57.

[8] OPAL Coll., G. Alexander et al., Phys. Lett. B370 (1996) 185.

[9] DELPHI Coll., P. Abreu et al., Z. Phys. C65 (1995) 555.

[10] DELPHI Coll., P. Aarnio et al. Nucl. Instr. and Meth. A303 (1991) 233.

[11] DELPHI Coll., P. Abreu et al., Nucl. Instr. and Meth. A378 (1996) 57.

[12] C. Peterson et al., Phys. Rev. D27 (1983) 105.

[13] T.Sjöstrand, Comp. Phys. Comm. 28 (1983) 227.

[14] JADE Coll., W.Bartel et al., Z. Phys. C33 (1986) 23;

JADE Coll., S.Bethke et al. Phys. Lett. B213 (1988) 235.

[15] S. Catani et al., Phys. Lett. B269 (1991) 432;

N. Brown, W.J. Stirling, Z. Phys. C53 (1992) 629.

[16] ALEPH Coll., D. Buskulic et al., Phys. Lett. B313 (1993) 535.

[17] G. Borisov and C. Mariotti, Nucl. Inst. Meth. A372 (1996) 181.

[18] M. Bengtsson and P. Zerwas, Phys. Lett. B208 (1988) 306.

[19] E. Accomando and A. Ballestrero, Comp. Phys. Comm. 99 (1997) 270. 